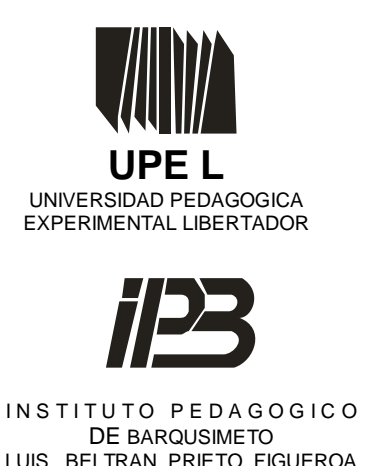

LUIS BELTRAN PRIETO FIGUEROA

\section{REVISTA}

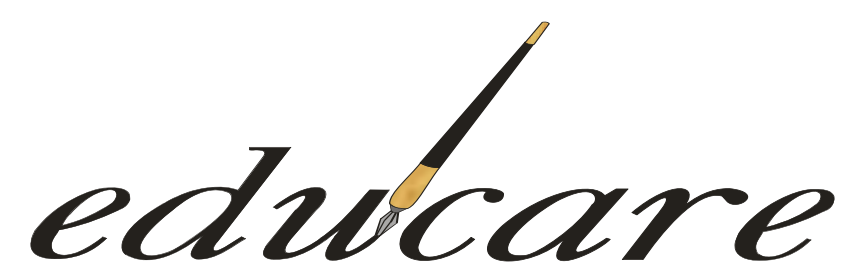

Órgano Divulgativo de la Subdirección de Investigación y Postgrado del Instituto Pedagógico de Barquisimeto "Luis Beltrán Prieto

Figueroa”

BARQUISIMETO - EDO. LARA - VENEZUELA

NUEVA ETAPA

FORMATO ELECTRÒNICO

DEPOSITO LEGAL: ppi201002LA3674

Volumen $17 \mathrm{~N}^{\circ} 2$

ISSN: 2244-7296

Mayo-Agosto 2013
IMAGEN CORPORATIVA COMO HERRAMIENTA DE GESTIÓN INSTITUCIONAL DE LA FACULTAD DE CIENCIAS ECONÓMICAS Y SOCIALES DE LA UNIVERSIDAD DEL ZULIA
CORPORATE IMAGE AS A TOOL FOR INSTITUTIONAL MANAGEMENT OF FACULTY OF ECONOMICS AND SOCIAL SCIENCE OF THE UNIVERSITY OF ZULIA

Graciela Perozo de Jiménez

Sorayda Rincón González

Mary Urdaneta

Universidad del Zulia

Venezuela 


\title{
IMAGEN CORPORATIVA COMO HERRAMIENTA DE GESTIÓN INSTITUCIONAL DE LA FACULTAD DE CIENCIAS ECONÓMICAS Y SOCIALES DE LA UNIVERSIDAD DEL ZULIA ${ }^{1}$
}

\author{
CORPORATE IMAGE AS A TOOL FOR INSTITUTIONAL MANAGEMENT OF FACULTY \\ OF ECONOMICS AND SOCIAL SCIENCE OF THE UNIVERSITY OF ZULIA
}

INVESTIGACIÓN

Recibido: 04-12-12

\author{
Graciela Perozo de Jiménez* \\ Sorayda Rincón González** \\ Mary Urdaneta**** \\ Universidad del Zulia \\ Venezuela
}

Aceptado: 12/06/2013

\section{RESUMEN}

El presente estudio, tuvo como objetivo fundamental determinar la Imagen Corporativa como Herramienta de Gestión Institucional de la Facultad de Ciencias Económicas y Sociales de la Universidad del Zulia. La metodología fue descriptiva, basada en el diseño no experimental, de tipo transversal descriptivo. Se realizó un muestreo no Probabilístico, utilizándose una muestra de participantes voluntarios, con un enfoque de campo, aplicándose la técnica de la encuesta. Entre los resultados vinculados con el conocimiento brindado por la FCES respecto a las actividades académicas que ofrece a la comunidad, se observó una alta presencia entre la comunidad consultada, pero en el caso de si la forma en darse a conocer en la comunidad es clara y precisa, el 53\% reflejo ausencia, aspecto importante a tomar en cuenta. Se concluye que se deben generar procesos comunicacionales para proyectar la imagen corporativa de la FCES, como elemento estratégico, para lo cual será necesario tener una visión global de la comunicación en el seno de las universidades y la relevancia que éstas tienen en el desarrollo del país.

Palabras Clave: Imagen, Comunicación, Gestión universitaria

\begin{abstract}
The present study had as main objective to determine the corporate image as a Tool for Institutional Management, Faculty of Economics and Social Sciences of the University of Zulia.

The methodology was descriptive, based on nonexperimental design, descriptive transversal. A nonprobabilistic sampling was carried out, using a sample of volunteer participants, with a focus on field, applying the technique of the survey. Outcomes related to knowledge provided by the FCES regarding academic activities offered to the community, a high presence in the community consulted was observed, but in case if the way to get noticed in the community is clear and precise, 53\% reflected absence, important aspect to consider. We conclude that communication processes must be generated to project the corporate image of the FCES, as a strategic element, it means it will be necessary to have a global view of communication within universities and the relevance they have on the country's development.
\end{abstract}

Keywords: Image, Communication, university management.

\footnotetext{
${ }^{1}$ Este artículo corresponde al Proyecto de Investigación Imagen Corporativa como Herramienta de Gestión Institucional de la Universidad del Zulia financiado por el CONDES

* Economista. Magíster en Gerencia de Empresa Mención: Mercadeo (LUZ). Doctora en Ciencias Gerenciales. URBE. Docente Titular de la Escuela de Administración y Contaduría. Investigadora del Centro de Estudios de la Empresa de la Facultad de Ciencias Económicas y Sociales de LUZ. Acreditada al Programa de Promoción al Investigador (PPI) Nivel I de la ONCTI. grajimenez@ cantv.net

** Socióloga. Magíster en Planificación y Gerencia de C y T (LUZ) Doctora en Ciencias Gerenciales. URBE. Docente Titular de la Escuela de Sociología de LUZ Investigadora del Centro de Estudios de la Empresa de la Facultad de Ciencias Económicas y Sociales de LUZ. Acreditada al Programa de Promoción al Investigador (PEI) de la ONCTI sorarincon@ hotmail.com

*** Lic. En Contaduría Pública, Magíster en Gerencia de Empresas. Mención Finanzas (LUZ) Docente Asociado de la Escuela de Administración y Contaduría. Investigadora del Centro de Estudios de la Empresa de la Facultad de Ciencias Económicas y Sociales de LUZ. Acreditada al Programa de Promoción al Investigador (PEI) de la ONCTI maryudta@gmail.com
} 


\section{INTRODUCCIÓN}

Los cambios en la sociedad, la expansión de los mercados, la globalización, la calidad y la competitividad son algunos de los mayores retos que deben enfrentar las instituciones de educación superior. Ante estos retos la nueva visión organizacional de las casas de estudios superior debe estar sustentadas no solo en la producción y preparación de sus profesionales sino incluir en sus principios básicos la imagen, la comunicación, la cultura, la identidad como ejes de acción ya que estos aspectos constituyen el sistema nervioso central de todos los procesos dinámicos de una institución moderna.

De acuerdo a Carrillo (2011) se espera de las universidades la creación de nuevos espacios integradores para las acciones de la educación superior y el establecimiento de nuevos entornos de comunicación que generen nuevas formas de interacción entre los miembros de la comunidad y con la ciudadanía en general.

Es así, como las universidades enfrentan grandes desafíos en todas partes del mundo, impulsados por los cambios en las sociedades orientados a satisfacer las presiones de la globalización económica y a los procesos de integración y en la búsqueda de mayores niveles de eficiencia, calidad para elevar la coherencia en los sistemas nacionales de educación superior.

Según Álvarez y Maldonado (2009) la universidad en este nuevo milenio está afrontando numerosos cambios, dilemas y retos, y debe prepararse para enfrentarlos conservando un pensamiento utópico. En este contexto, todos los actores que hacen vida en ella deberían insistir en el derecho a ensanchar y soñar con el futuro. Un pensamiento utópico nos permite imaginar una universidad diferente que guíe las actividades de los docentes, alumnos, administrativos y autoridades, en aras de lograr la universidad soñada por todos.

De igual manera, en Venezuela, la crisis que atraviesan las instituciones públicas se evidencia en diversos aspectos, entre ellos, la falta de credibilidad en el ejercicio de la administración pública, así como el deterioro de la imagen de sus líderes e instituciones. Como plantea Black (2004), la imagen corporativa es el capital de prestigio de una organización, la percepción de sus públicos (internos y externos) con respecto al compromiso de la misma lo que determina su confiabilidad, credibilidad y reputación, siendo un activo frágil e intangible. 
Una vez presentado a la UNESCO (1978) el informe sobre las nuevas tendencias en gerencia de la comunicación, las universidades latinoamericanas comienzan a incluir la comunicación organizacional en sus planes estratégicos, haciendo énfasis en la comunicación y promoción interna y externa para garantizar la supervivencia y posicionamiento en el entorno. En consecuencia, los planes comunicacionales de las organizaciones están íntimamente ligados a las actividades generales de estas, al momento que viven y desde el punto de vista más general a los procesos por medio de los cuales la organización se posiciona por medio de su imagen corporativa.

En todas las organizaciones existen diferentes formas de comunicarse, independientemente que la misma sea pública o privada, o se dedique a ofrecer bienes o servicios. Para realizar dicha labor con éxito hay que entender la imagen corporativa como una herramienta de gestión valiosa la cual permite mantener una relación eficiente con sus públicos objetivo internos y externos además de eso también se debe estudiar y conocer, a fin de que la institución pueda generar políticas y estrategias comunicacionales cónsonas con el quehacer de la institución.

Es de todos conocido la crisis por la que atraviesan las universidades venezolanas amenazadas por factores internos y los efectos que en ella genera el ambiente externo como la creciente competitividad nacional e internacional, el crecimiento tecnológico, el desarrollo de la globalización en los procesos mundiales y las nuevas tendencias características de una sociedad emergente, que demandan de las universidades una nueva visión para adaptarse a los cambios y enfrentarse a la incertidumbre, impulsando el desarrollo económico y social del país. Asimismo, cada día se evidencia más los problemas en estas instituciones generados por las limitaciones de recursos para su funcionamiento, decretos que amenazan su autonomía, además de la falta de vinculación de estas con la sociedad en general.

Como es sabido, la imagen y la comunicación son elementos fundamentales no solo para las empresas privadas, sino también para las instituciones públicas y por ende para las organizaciones sin fines de lucro. Es por eso que en las universidades, de acuerdo con Trelles (2005) los procesos comunicacionales adquieren una gran relevancia porque las mismas no pueden prescindir de éstos para responder a su razón social. Dado que son instituciones vinculadas a la formación, construcción y socialización del conocimiento, por lo que la 
comunicación se convierte en un punto de partida imprescindible para este tipo de instituciones.

Es así como, para establecer la Imagen Corporativa de las Instituciones y en este caso en particular de la Facultad de Ciencias Económicas y Sociales (FCES) de la Universidad del Zulia, se requiere estudiar las actuaciones de estas organizaciones en el plano funcional y operativo orientadas a visualizar su: Imagen. Es por ello, que el objetivo del presente artículo se centró en: Establecer las necesidades en materia de Comunicación Corporativa de la organización objeto de estudio.

Dentro del ámbito universitario, se han realizado estudios referidos a la imagen de la Universidad del Zulia, en las cuales se evidencian aspectos que invitan a reflexionar y a plantear una propuesta alternativa de un plan de imagen y comunicación corporativa, basada en la identidad propia, para la FCES, que adaptada a la misma permitirá un exitoso comportamiento corporativo como herramienta de gestión institucional. La revisión de estos antecedentes de investigación, referidos a estudios de la imagen y/o comunicación de la Universidad del Zulia, evidencian aspectos fundamentales sobre las fallas que la institución tiene, en cuanto a la estrategia de proyección de su Imagen a través de comunicación corporativa.

Entre los antecedentes estudiados se destacan:

a) La investigación" imagen corporativa en la Universidad del Zulia" cuyos autores plantearon que una gran conclusión de esta investigación se deriva de la ineficiencia de los medios universitarios para difundir oportuna y verazmente sus actividades, programas y decisiones, con lo cual se comete un grave error en la construcción de la imagen corporativa de la institución. (Rincón, Graterol, Díaz 2003)

b) Otros estudios considerados sobre el tema sería el de Miquilena (2002) titulada, "Imagen corporativa de Instituciones de Educación Superior según el Plan de Desarrollo Estratégico", el cual concluyo como evidencia significativa que la imagen corporativa de la Universidad del Zulia a partir del modelo de comunicación que aparece en el plan de desarrollo estratégico, dista mucho del deber ser, y por ende trasmite una débil imagen institucional

c) Igualmente, otra evidencia documentada por Sáez (2003) en su investigación titulada "Estrategias de mercadeo para reposicionar la imagen institucional en los 
estudiantes de las Universidades Públicas Nacionales en el Municipio Maracaibo”, concluye que los resultados expuestos, permiten analizar la percepción de la imagen institucional, entre estudiantes activos de LUZ. Dichos resultados reflejan como los estudiantes tienen una percepción favorable de la institución en lo referente a calidad de la enseñanza y calidad del egresado, pero en aspectos como: el ambiente institucional, los servicios administrativos y estudiantiles, la proyección de LUZ hacia la comunidad y los recurrentes problemas presupuestarios que generan una alta frecuencia de paros y huelgas, la percepción del estudiantado incluido en la muestra es desfavorable.

d) Es importante resaltar otro estudio realizado sobre la Gestión comunicacional, sin considerar específicamente como objeto de estudio una institución universitaria en particular como el de: Ochoa (2004) en su tesis doctoral "La comunicación en el proceso de Transformación Universitaria" la cual tuvo como propósito general establecer los factores vinculantes de la comunicación social organizativa como estrategia de transformación en la educación superior, en donde empleo para ello una metodología descriptiva de campo, bajo una perspectiva cualicuantitativa.

e) también se debe considerar el estudio realizado por Suárez (2009) "Gestión Comunicacionales en las Unidades de Información y Comunicación de la Universidad del Zulia en el que demuestra que la Universidad del Zulia", en la que demuestra que LUZ, no realiza una adecuada gestión comunicacional en cada una de sus unidades de información y comunicación, agregando que más de la mitad de los comunicadores de la institución no conocen la política Comunicacional.

La investigación pretendió abordar el enfoque gerencial de la comunicación, su proceso tecnológico, la imagen de la universidad y los aportes de la comunicación en la transformación de estas casas de estudio; lo que permitió concluir que el modelo gerencial común es el mejoramiento continuo, por la falta de la gestión de los medios en cada institución, para orientar la acción comunicativa hacia una estrategia de pertinencia y relevancia social. 


\section{ALGUNAS REFLEXIONES TEÓRICAS}

\section{Comportamiento corporativo en la gestión universitaria}

El comportamiento corporativo de las universidades, deben estar basados en un modelo de gestión en el plano funcional y operativo de los procesos productivos de acuerdo a nuestro criterio basados en lo que representa la Imagen - la Comunicación - la Identidad corporativa. Por lo tanto, el comportamiento de las organizaciones debe estar reglamentado sobre la base de todas las expresiones en la que se mezcla su imagen, en función a establecer todas las normas operativas a las que debe atenerse la institución a fin de que la imagen corporativa se convierta en un elemento definitivo de diferenciación y posicionamiento frente a la sociedad.

Por otra parte, la gestión universitaria en sus múltiples dimensiones y en el contexto de operaciones y acciones que debe desarrollar para estar a la par de las exigencias externas e internas, se van haciendo más complejas y en algunas ocasiones en detrimento de sus respuestas, las cuales dejan de ser oportunas y eficientes es por ello que debe asumir estilos dinámicos de gerencia tomando en cuenta las nuevas tendencias, sistematización de procesos, así como las herramientas de gestión contemporáneas para ir a la par con las exigencias del mundo globalizado. (Ferrer y Pelekais, 2004)

Las organizaciones requieren ser administradas eficientemente, a fin de garantizar la coordinación de los esfuerzos individuales en la búsqueda de promover la excelencia entre todas las personas que forman parte de las mismas

Por lo tanto, se debe identificar lo que significaría la gestión en toda organización o institución según sea el caso, como lo definen Ivanevich, Lorenzi, y Skinner, (1997) "la gestión como el proceso emprendido por una o más personas para coordinar actividades laborales de otras personas con la finalidad de lograr resultados de alta calidad que cualquiera otra persona, trabajando sola, no podría alcanzar". (p.12)

Los autores antes mencionados, plantean que la gestión debe considerarse desde diferentes aspectos, en principio, la gestión es un proceso que comprende determinadas funciones y actividades laborales que los gestores deben llevar a cabo a fin de lograr los objetivos de la empresa (ibidem). 
Asimismo la gestión como disciplina, implica que se trata de un cuerpo acumulado de conocimientos susceptibles de aprendizaje mediante el estudio, conformada por principios, conceptos y teorías. De igual manera la gestión y las personas, se refiere a los "gestores" que tiene a su cargo el proceso de gestión, asumiendo la responsabilidad principal por la realización del trabajo en una organización.

Es importante resaltar lo que significa para las organizaciones la comunicación corporativa como instrumento de gestión, como lo consideran Koonttz, y Weihrich, (1998), al plantear que la comunicación es esencial para todas las fases del proceso administrativo, por dos razones, primero, integra las funciones administrativas, por ejemplo los objetivos establecidos en la planeación se comunican a otros con el propósito de idear la estructura organizacional más indicada. De igual modo, el liderazgo eficaz y la creación de un entorno conducente a la motivación dependen de la comunicación. Es así, como la administración es posible por la comunicación.

Segundo, el propósito de la comunicación, de acuerdo a los autores es enlazar a la empresa con su ambiente externo, en el que se encuentran muchos de los reclamantes, por ejemplo no se puede olvidar nunca que los clientes, razón de existir prácticamente de todas las empresas, se hallan fuera de la compañía.

Es por medio del sistema de comunicación, que se identifican las necesidades de los clientes, que permiten a las organizaciones brindar los productos y servicios y obtener las ganancias. De la misma manera, es mediante una eficaz comunicación que las organizaciones se ponen al tanto de la competencia, otras posibles amenazas y factores restrictivos. (Koontz y otros, 1998)

Todo lo antes expuesto, permite evidenciar el objetivo de la presente investigación que es determinar la imagen corporativa como herramienta de gestión institucional de la Facultad de Ciencias Económicas y Sociales de la Universidad del Zulia, a fin de generar lineamientos comunicacionales que conlleven a consolidar la imagen institucional de las mismas.

\section{Imagen Corporativa en las Instituciones}

Para la organización, la imagen corporativa es un elemento estratégico y un principio de gestión de allí que está relacionada con las operaciones, a fin es crear determinadas impresiones en los públicos, siendo la imagen un reflejo de la identidad cuyo objetivo final es 
determinar la actitud de los públicos objetivos en un sentido que sea positivo para las instituciones. (Villafañe, 1993)

La imagen corporativa, es el conjunto de principios y técnicas, que tienen de ciencia y arte, que permiten diferenciar y consolidar, entre toda la vasta competencia a las personalidades o grupos con verdadera esencia y capacidad de permanencia. (Pizzolante, 1999). Según el autor, no todo individuo o empresa está en condiciones directa e inmediata de construir una estructura corporativa que le otorgue reputación (imagen), más allá de la calidad de los productos o servicios y la capacidad operativa, la condición previa está en realizar un análisis crítico cualitativo de su estructura interna y estar consciente de las amenazas y oportunidades externas. La imagen no puede verse o pensarse como una máscara externa adaptable convenientemente, sino como la prolongación de su estructura interna y de su forma de actuar.

Es así, como Pizzolante (ob.cit.) refiere que "los principios, normas y valores de la empresa, su trayectoria y el comportamiento de su personal, modelan lo que se ha llamado cultura corporativa" a su vez plantea que la "forma de ser y hacer empresa constituye la identidad corporativa" la cual, "al ser proyectada crea un fenómeno de opinión pública denominada Imagen”. (p. 3)

Es a través de esta teoría en principio, que se va a sustentar la presente investigación, sobre, la imagen corporativa a través de un sistema de identidad y cultura corporativa. Como lo plantea Pizzolante (ob.cit.):

La imagen es una estructura mental construida por el ser humano, la cual como todas las estructuras seguras, debe tener cimientos fuertes, productos de un buen diseño. Fundaciones apuntaladas en valores y normas, cultura corporativa que modela la identidad de la empresa y permite edificar sobre ella un arquitectónico diseño de columnas y vigas, que se convierten en estrategias y mensajes para apuntalar la imagen corporativa. (p.1)

La permanente y ejemplar actitud de algunas empresas constituye una firme evidencia acerca de cómo consideran importantes sus imágenes, y tal vez es la mejor respuesta a los que, por desconocimiento e inseguridad, cuestionan en este momento el valor de la imagen. Para Madia de Souza (1995), "la imagen es el mejor seguro de vida que empresa alguna puede 
tener", así mismo refiere "La imagen es lo que las personas - públicos decisivos y relevantescreen que la empresa es". (p.207)

De igual manera, se va a considerar la definición de Dowling, citado por Van Riel (1997) "una imagen es el conjunto de significados por lo que llegamos a conocer un objeto, y a través del cual la persona lo describen, recuerdan y relacionan. Es el resultado de la interacción de creencias, ideas, sentimientos, e impresiones que sobre un objeto tiene una persona" (p.77)

El interés en el estudio de la imagen de las organizaciones radica, en la suposición de que una imagen positiva es el requisito previo básico para establecer una relación comercial, directa o indirecta con diferentes públicos objetivo.

En otro orden de ideas, se plantea que la imagen se forma como resultado de los estímulos que un sujeto recibe de un objeto, si estos consiguen impactar y llamar la atención, él les dará un significado, para luego aceptarlos con lo cual la información produce el efecto deseado por la fuente. Finalmente el individuo se centrará en el posible almacenamiento de los estímulos a largo plazo de forma organizada (Van Riel, 1997)

Considerando además, los planteamientos de Scheinsohn, (1998) quien sostiene que la imagen corporativa es conceptualizada como un bien, que si bien, es manipulado por la empresa desde su identidad, está en poder de los públicos, pues son ellos los que constituyen la imagen de ese sujeto social, adaptándola a un sistema de creencias. En síntesis la imagen corporativa la construyen los públicos, más allá de lo que la empresa planifica. Por lo tanto, se define la imagen corporativa, como la síntesis mental que los públicos elaboran acerca de una determinada empresa, a partir de elementos muy heterogéneos como los signos visuales, cultura, experiencia, percepción, sensaciones, memoria, entre otras influencias.

Estos planteamientos del autor precitado, sobre lo que define la imagen corporativa, deben servir de base para las instituciones universitarias al considerar aquellos elementos que quieran o deban proyectar de sus instituciones, basadas en elementos relevantes como la vasta experiencia que tiene muchas de ellas sustentadas por un prestigio que la cultura sustenta en ellas como el caso de la Universidad del Zulia.

La imagen corporativa, en tanto construcción de los públicos, se produce a partir de la percepción de los actos cotidianos que la empresa lleva a cabo, no solo de los mensajes 
explícitos y voluntarios que ella propone emitir, sino además de aquellos otros que emite tal vez sin considerar. (Scheinsohn, ob.cit.)

Por lo que es de relevancia significativa, entender que la imagen corporativa la construyen los públicos y las organizaciones son las responsables de la imagen que los públicos elaboran acerca de ellas, aunque éstas no gestionan su imagen de manera directa sino indirectamente a través de las comunicaciones y promociones que desarrollan, en la búsqueda de estructurar en el público objetivo un determinado resultado.

Es importante considerar otros aspectos sobre la imagen como los planteados por Costa (2003) quien expone que tener una imagen es tener una imagen mental. Ello posee un doble significado. En primer lugar supone la aptitud natural del individuo para reconstruir, en otro momento una representación mental de alguien o de algo, implicado en esta reconstrucción los valores de forma y papel. En segundo lugar, y correlativamente con esta aptitud, tener una imagen implica al mismo tiempo que el individuo receptor se halla expuesto a los efectos sicológicos de esta imagen. De esta manera se define la imagen como la representación mental de una empresa que tiene capacidad de condicionar y determinar, incluso, las actitudes de un grupo social en relación con esta empresa

De allí que de acuerdo a esa postura, tener una imagen implica, la existencia de un proceso que abarca desde la percepción del dato significativo y su memorización, hasta sucesivas percepciones y asociaciones de ideas, y la configuración de un estereotipo, el cual rige finalmente determinadas conductas. Estos aspectos deben ser considerados por las instituciones a fin de ir desarrollando la percepción de la imagen que las mismas quieren proyectar convirtiéndose en elementos de memorización por parte de los públicos a fin de condicionar los mismos sobre los aspectos que las organizaciones quieran positivamente introducir en las mentes de los usuarios de su entorno, tanto interno como externo.

Otro autor importante a tomar en cuenta dado sus conceptos actualizados es Garrido, (2004), quien define la imagen de empresa como "el resultado que se produce en el auditorio tras la interpretación de los elementos disponibles para su campo perceptivo en el discurso corporativo" (p.194). El autor propone que la imagen será una construcción mental evolutiva que se aloja en el consciente y subconsciente de los públicos, que tenderá a determinar sus actitudes y opiniones respecto de la compañía. 
En esta definición se observa la coincidencia entre los autores Costas (2003) y Garrido (2004) acerca de las actitudes que asumen los públicos sobre las organizaciones en función a la imagen mental que estos crean en sus mentes, opiniones, percepciones, memorizaciones, sobre las mismas, ya que las mismas serán evaluadas, de acuerdo a los ejes comunicacionales que la empresa proponga, dándole a los auditorios los conceptos que ellos otorguen mayor relevancia y valor relativo.

Para Garrido (2004), las expectativas que los públicos se crean a partir de los mensajes consumidos por acción propia o por iniciativa de la empresa, serán evaluadas como cumplidas o como no cumplidas a raíz de los encuentros del público con el sujeto emisor, lo que producirá La confirmación o rechazo de sus antecedentes y creencias, a través de una acción comparativa con la realidad "conocida".

Se infiere entonces, en la postura de Garrido (ob.cit.) que debe tomarse en cuenta la idea de la imagen como una concepción general, puede ser comprendida como el resultado de un estado de apariencia; por el contrario, en el caso de la empresa que requiere y se ve obligada a una verificación constante de sus públicos objetivo, deberá ser más el trabajo constante y sustancial de la empresa, que sólo el producto de la apariencia, para conseguir una proyección sustancial en la sociedad y efectos positivos a largo plazo, que debe ser la meta de todas las organizaciones y en este caso de las instituciones universitarias, no podrá conseguirse eficientemente al margen de la comunicación estratégica o corporativa.

Todo este planteamiento de los autores antes citados, nos lleva a establecer concordancia con lo establecido por Gómez y Villalobos, (1998) dado que su concepto de imagen corporativa es la percepción que la gente tiene y no el ente cree debe tener. Este último es el objetivo a lograr.

En resumen, la imagen no es más que la opinión que se alberga sobre una organización después de haber conocido su identidad y ver su actuación en el ambiente donde desarrolla sus actividades. Esta imagen puede generarse por mecanismo de inercia, pero también puede ser planificada por unidades especializadas dentro de la organización o por empresas consultoras.

En este sentido, las investigadoras plantean la necesidad creciente de gestionar la imagen corporativa de las organizaciones basadas en la comunicación y la identidad corporativa de las mismas, de forma planificada y no dejarla solo al azar de la percepción y 
expectativas que de ellas tienen sus públicos objetivo, coincidiendo con los planteamiento de los autores venezolanos Gómez y Villalobos (ob.cit.) quienes establecen que "la gerencia de la imagen corporativa u organizacional es, naturalmente lo más aconsejable”(p.15)

El recurso gerencial y el estilo que se aplique van a ser determinante. El cumplimiento de los objetivos y metas programadas por las empresas en este caso por las instituciones, definen en buena parte, su perfil de imagen. La Imagen corporativa hay que verla como la resultante de un esfuerzo planificado, compartido, sistemático y coherente para hacer que la actividad organizacional se desarrolle eficientemente y así generar bienestar colectivo, siempre con el soporte de un buen estructurado sistema de comunicaciones. Otro aspecto preponderante a tomar en cuenta y como complemento a lo anteriormente dicho, surge de la necesidad de considerar a los directivos como elementos de identificación de las organizaciones, ya que su buena o mala actuación influirá en el perfil de la imagen que el público se forme de ella, esto es muchos más notorio en la administración pública, no escapándose de ello las autoridades universitarias a todos los niveles.

De esta manera la imagen no es más que la necesidad que se tiene de constituir, mantener o reforzar la verdadera personalidad organizacional, tanto en el área cultural como en lo visual. Es un valor agregado cuyo desarrollo será la base para su proyección, pero es importante que esa gestión se fundamente en una adecuada comunicación dirigida a públicos específicos, por lo que se considera es una tarea donde entra en juego tres elementos insustituibles: la organización, la comunicación y los públicos.

\section{Comunicación Corporativa en las Organizaciones}

La comunicación corporativa se compone de un gran número de elementos, tanto internos como externos, que desarrollándolos eficientemente, se convierten en la plataforma de proyección de la imagen de las organizaciones. Es así como la comunicación corporativa se define como el conjunto de mensajes que una institución proyecta a un público determinado a fin de dar a conocer su visión, misión, y lograr establecer una empatía entre ambos. Por lo que la misma deber ser dinámica, planificada y concreta constituyéndose en una herramienta de dirección basada en una retroalimentación constante.

La comunicación Corporativa para Van Riel (1997) es un instrumento de gestión por medio del cual toda forma de comunicación interna y externa conscientemente utilizada, está 
armonizada tan efectiva y eficazmente como sea posible, para crear una base favorable para las relaciones con los públicos de los que la empresa depende. (p. 26)

Para este autor, la comunicación corporativa surge principalmente de la práctica comercial, pero la necesidad de profundizar en las perspectivas, y de un apoyo completo para resolver los problemas por personas con conocimiento práctico a diario, no necesariamente se complementan en orientación sólo a lo comercial.

La comunicación corporativa es una estructura en la que todos los especialistas en comunicación (marketing, comunicación organizativa y de gestión) forman la totalidad del mensaje organizativo, y de este modo ayudan a definir la imagen corporativa como el medio que lleva a mejorar la realización corporativa. Aquí se resalta el significado que se le concede al término Comunicación Corporativa, el cual es reconocido internacionalmente, y aparece regularmente en la literatura, considerándolo en un sentido figurado relacionado al todo.

Las ideas básicas a la comunicación corporativa son relevantes, tanto para las empresas privadas y las organizaciones no lucrativas, dada la necesidad creciente de crear y mantener una imagen favorable para sus públicos objetivo. Si se parte del concepto del autor Van Riel, (ob.cit.) sobre comunicación corporativa, donde establece que toda comunicación interna y externa eficazmente utilizada crea una base favorable para las relaciones con los públicos, es de relevancia primordial identificar esos públicos objetivo o mercados objetivos, a través de sus características y necesidades.

Por otra parte Van Riel (ob.cit) señala, la comunicación corporativa es principalmente el enfoque que varios especialistas en comunicación, dentro de un marco estratégico, pueden aportar para coordinar las funciones comunicacionales, se utilizan tres formas principales de comunicación en las organizaciones: la comunicación de dirección, la comunicación de marketing (Mercadeo) y la comunicación organizativa.

Actualmente se considera una integración entre estas tres formas de comunicación a fin de evitar el peligro de una comunicación fragmentada, por lo cual se busca un esfuerzo integral de comunicación corporativa para conseguir mayor coherencia entre toda forma de comunicación interna y externa, contribuyendo de esta manera positivamente a una imagen favorable de cada elemento individual en la organización, quien a su vez, trabaja para apoyar la correcta actuación general de la institución. 
El esfuerzo por conseguir una mayor coordinación en la comunicación total en las organizaciones, surge de los especialistas en el campo del diseño, y luego en el campo de la comunicación de marketing. Como lo plantea Van Riel (ob.cit.), desde la perspectiva de la comunicación corporativa toda forma de comunicación debe estar considerada para la potencial orquestación hacia la coherencia total. La filosofía base que fundamenta esta idea podría describirse como la que dirige la política de comunicaciones de empresa dentro del triángulo "estrategia corporativa - identidad corporativa - imagen corporativa“.

Es así como, los representantes de las diversas especialidades en comunicación desarrollan en conjunto los "puntos de partidas comunes", derivados directamente de la estrategia de comunicación elegida, a su vez consecuencia de la identidad corporativa real y de la imagen de la empresa (Perozo, 2002)

Por su parte Gómez y Villalobos (ob.cit.) plantean que la comunicación corporativa u organizacional, debe ser entendida como un sistema de distribución de mensajes claves, que para su ejecución requiere un proceso de investigación, a fin de establecer sus antecedentes históricos, la misión, los objetivos, y definir políticas, estrategias y acciones, que se implementaran en el área de la institución que tenga bajo su responsabilidad la ejecución del plan operativo de comunicaciones.

Todo esto lleva a establecer de acuerdo a Gómez y Villalobos (ob.cit) que los “propósitos de la comunicación corporativa son lo de eliminar la incertidumbre, fortalecer la imagen, eliminar la desproporción entre la calidad y cantidad de información que se emita, aprovechar los recursos, eliminar las discordancia entre emisor y receptor por causa del código usado, estimular un buen clima organizacional y servir de balance entre la organización y sus público”. (p 16)

\section{La Gestión de la Comunicación Corporativa}

Según Scheinsohn (ob.cit.) la comunicación debe ser entendida como una función eminentemente estratégica, lo cual implica ejecutar un plan global y totalizador, donde la empresa sea asumida como un todo, un sistema total, es decir, lo que técnicamente se denomina un emergente sistémico. Para el autor, la comunicación corporativa, es la herramienta de gestión por excelencia, coincidiendo con ello con los planteamientos de Van 
Riel (ob.cit.), cuando establece que la comunicación corporativa es un instrumento de gestión valiosa para su relación con los públicos.

Lo que plantea Scheinsohn (ob.cit.) es que

La comunicación corporativa, intenta hacer es tratar de obtener una mayor gobernabilidad sobre el amplio campo de emisión, reconociendo en lo posible, los mensajes involuntarios con anticipación a la emisión efectiva; y si los reconoce con posterioridad a la emisión, intervenir para: capitalizar el rédito de lectura en caso que sea favorable a la estrategia, o bien, en caso de resultar negativos, atenuar los efectos indeseables. (p.169)

Se establecen distintas áreas de gestión comunicacional, partiendo del hecho que la clasificación que el autor presenta se corresponde con el concepto de comunicación corporativa como temática de intervención de comunicación estratégica, de igual manera se expone que no todas las empresas deben, pueden y/o quieren gestionar la totalidad de las áreas definidas, dado que cada empresa, basada en sus necesidades y problemática específica, podrán configurar otras áreas de gestión.

Las áreas de gestión identificadas, se corresponde a una clasificación más operacional que las clasificaciones tradicionales, por lo cual define diez áreas de gestión de la comunicación corporativa: Comunicación de Diseño, Comunicación de Marketing, Relaciones Institucionales y con la Comunidad, Relaciones Gubernamentales, Comunicación Financiera, Comunicación Business to Business, Relaciones con el Periodismo, Comunicación Interna, Cybercomunicaciones y Multimedia, Administración de Datos e Indagaciones.

Todas estas áreas de intervención son las que el autor denomina el Diamante de Gestión Comunicacional, que es un instrumento mediante el cual la comunicación estratégica, lleva a cabo la gestión de la temática de intervención denominada Comunicación corporativa. (Scheinsohn, ob.cit.)

Para Garrido, (2004) significa definir la estrategia de comunicación como un marco ordenador que integra los recursos de comunicación corporativa en un diseño de largo plazo, conforme a objetivos coherentes, adaptables y rentables para la empresa.

La implementación de una estrategia de comunicación corporativa será motor de cambios en los modos de gestión de las comunicaciones de la empresa, la cual se traduce en acciones, tácticas y campañas, que ponen en acción los propósitos planteados a nivel de 
diseño, por parte de los encargados de la administración y gestión de la comunicación en la empresa.

Aun cuando las organizaciones son un ser comunicacional y económico por naturaleza, esto no asegura que sus recursos comunicacionales sean en general gestionados de forma eficiente, o que sus costos de comunicación con sus clientes se traduzcan en inversiones, como se ha observado en las instituciones universitarias estudiadas las cuales presentan varios problemas de gestión comunicacional como sería: Falta estructuras de comunicación, insuficiencias operacionales, carencia de orientación estratégica, responsabilidades diluidas y resolución inapropiadas entre otras. (Garrido, ob.cit.).

Ante estos problemas, se propone unos elementos básicos en la gestión de comunicación, que las organizaciones y/o instituciones deberán desarrollar de manera sistemática: Investigación; interpretación y Análisis; Diseño Estratégico; Dirección y Gestión; Integración y Evaluación y Control.

De allí, que tanto la investigación cuantitativa o cualitativa, como la capacidad de análisis de los profesionales para el diseño de estrategias y planes orientadores de la gestión a largo plazo de la comunicación de la compañía, cumplirán el objetivo al ser capaz de integrar los diversos instrumentos, soportes, canales, mensajes y actuaciones en torno a un objetivo común y sinérgico.

Los argumentos reflexivos presentados en referencia al planteamiento de autores como Van Riel (ob.cit.), Gómez y Villalobos, (ob.cit.), Scheinsohn, (ob.cit.) y Garrido, (ob.cit.), configuran en su conjunto una visión compartida al considerar la Comunicación Corporativa, como una herramienta relevante de gestión estratégica en las organizaciones.

\section{ARGUMENTACIÓN METODOLÓGICA}

Es importante resaltar entre los aspectos metodológicos, que el estudio se clasificó como una investigación aplicada ya que tiene como finalidad principal resolver un problema de la realidad; de igual manera, el estudio es descriptivo, basado en el diseño de tipo no experimental por cuanto la variable objeto de estudio no fue manipulada en ningún momento. Asimismo, considerando la dimensión temporal se puede inferir que fue de tipo transversal descriptivo porque tuvo como objeto ubicar, categorizar y proporcionar una visión del contexto en estudio en un punto determinado en el tiempo. 
Se realizó un muestreo no Probabilístico, por lo tanto el estudio sobre la imagen y comunicación corporativa en la Facultad de ciencias económicas y sociales (FCES) de La Universidad del Zulia (LUZ), fue una investigación de carácter descriptiva, para la cual se utilizó una muestra de participantes voluntarios, muy frecuentemente utilizada en las ciencias sociales, con un enfoque de campo.

La población de interés estuvo constituida por personal Académico Universitario, con dedicación exclusiva y categoría superior a instructor. Se determinó una muestra de treinta (30) sujetos, docentes de la FCES-LUZ en la ciudad de Maracaibo. La información primaria se obtuvo mediante el uso de la técnica de la Entrevista directa en los lugares de trabajos de los entrevistados.

\section{RESULTADOS DEL ESTUDIO}

Los resultados que a continuación se presentan y analizan son el producto de la investigación de campo; interpretando la data de manera general de los diferentes entrevistados.

Se van a demostrar ahora los resultados relacionados con la Dimensión Necesidades en Materia de Comunicación (Grafico1). En cuanto a si la FCES da a conocer las actividades académicas que ofrece a la comunidad, se puede visualizar que el $57 \%$ reflejó presencia y el $43 \%$ reflejó ausencia en cuanto a dar a conocer las actividades, si se considera si la forma en darse a conocer en la comunidad es clara y precisa el 53\% reflejó ausencia y el $47 \%$ reflejó presencia.

Contrastando el resultado con lo que plantea Van Riel, 1997, la comunicación debe estar armonizada tan efectiva y eficazmente como sea posible, para crear una base favorable para las relaciones con los públicos de los que la empresa depende. Lo que significa que aun cuando la FCES da a conocer las actividades académicas en un porcentaje mayor, la forma de proyectar la misma no es de forma clara lo que evidencia la necesidad de revisar la información proyectada a la comunidad. 


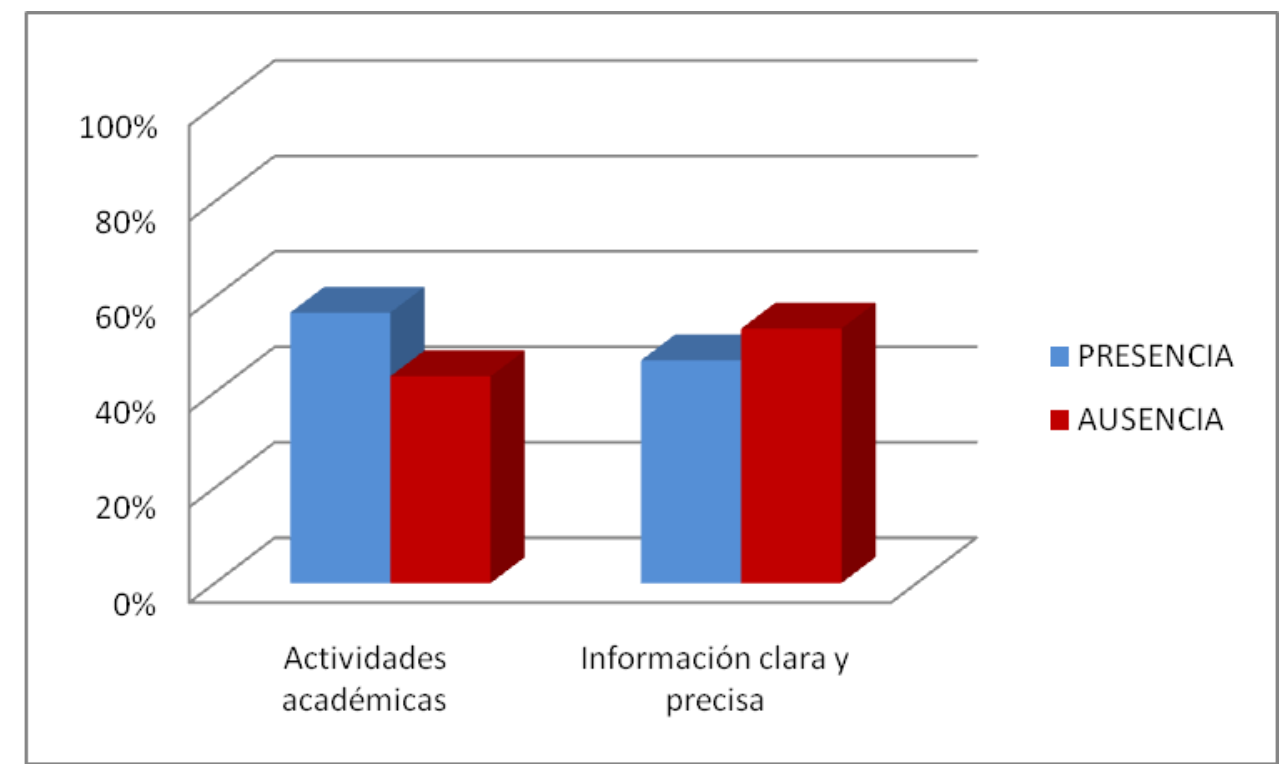

Fuente: Elaboración propia

\section{Gráfico 1.}

\section{Conocimiento de las Actividades Académicas}

Observando el Grafico 2, relativo a como los medios de comunicación difunde información acerca de la FCES los resultados reflejan que el 53\% observan presencia en los medios, en contra de un $47 \%$ que observa ausencia. Si se analiza el aspecto relativo a si las autoridades de la Facultad, mantiene una comunicación directa con su personal el $67 \%$ una tendencia alta, refleja ausencia en relación a la comunicación que se debería tener con el personal que considera un $33 \%$ de ausencia, aspecto muy relevante a tomar en cuenta a fin de generar los correctivos necesarios para mejorar este aspecto comunicacional.

Es así como Gómez y Villalobos (ob.cit.) plantean, que la comunicación corporativa, debe ser entendida como un sistema de distribución de mensajes claves, que para su ejecución requiere un proceso de investigación, a fin de establecer sus antecedentes históricos, la misión, los objetivos, para poder definir, políticas, estrategias y acciones, que se implementarán en el área de la institución en la ejecución del plan operativo de comunicaciones. 


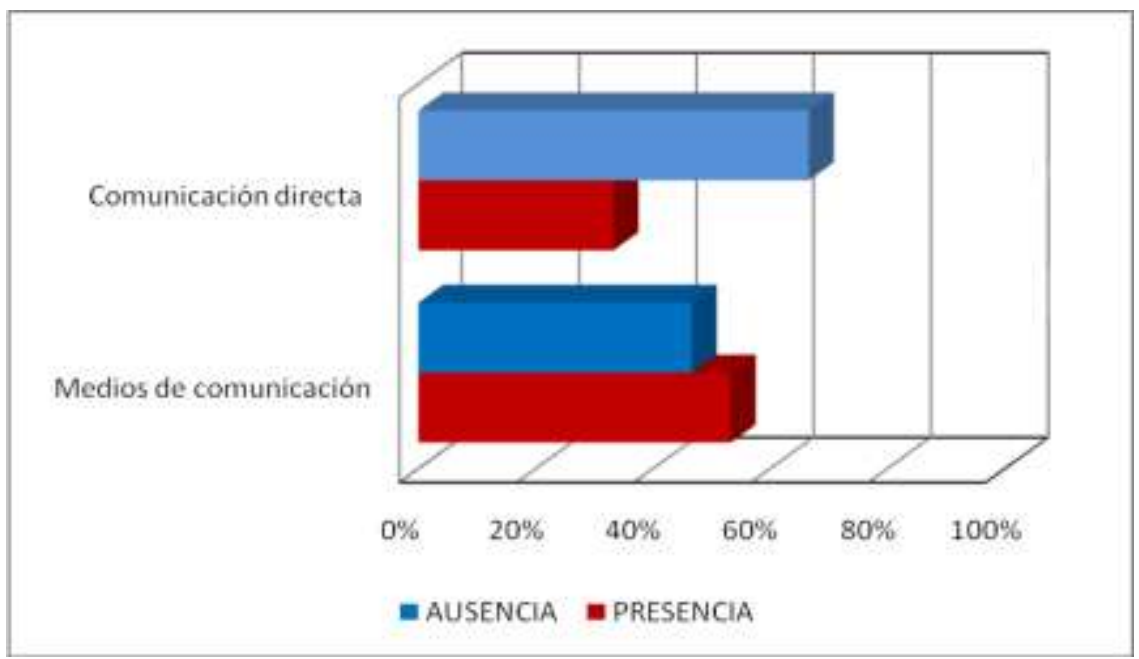

Fuente: Elaboración propia

\section{Gráfico 2.}

\section{Medios de Comunicación para difundir la Información}

De acuerdo con los resultados plasmados, en relación a la pregunta, a través de que medios el personal académico se entera de las actividades que desarrolla la FCES, se puede evidenciar en el Grafico 3, que un $60 \%$ reporta que es a través de la Internet, vía correos electrónicos, el 20\% la prensa, el 13 \% la radio y el 7\% la Televisión.

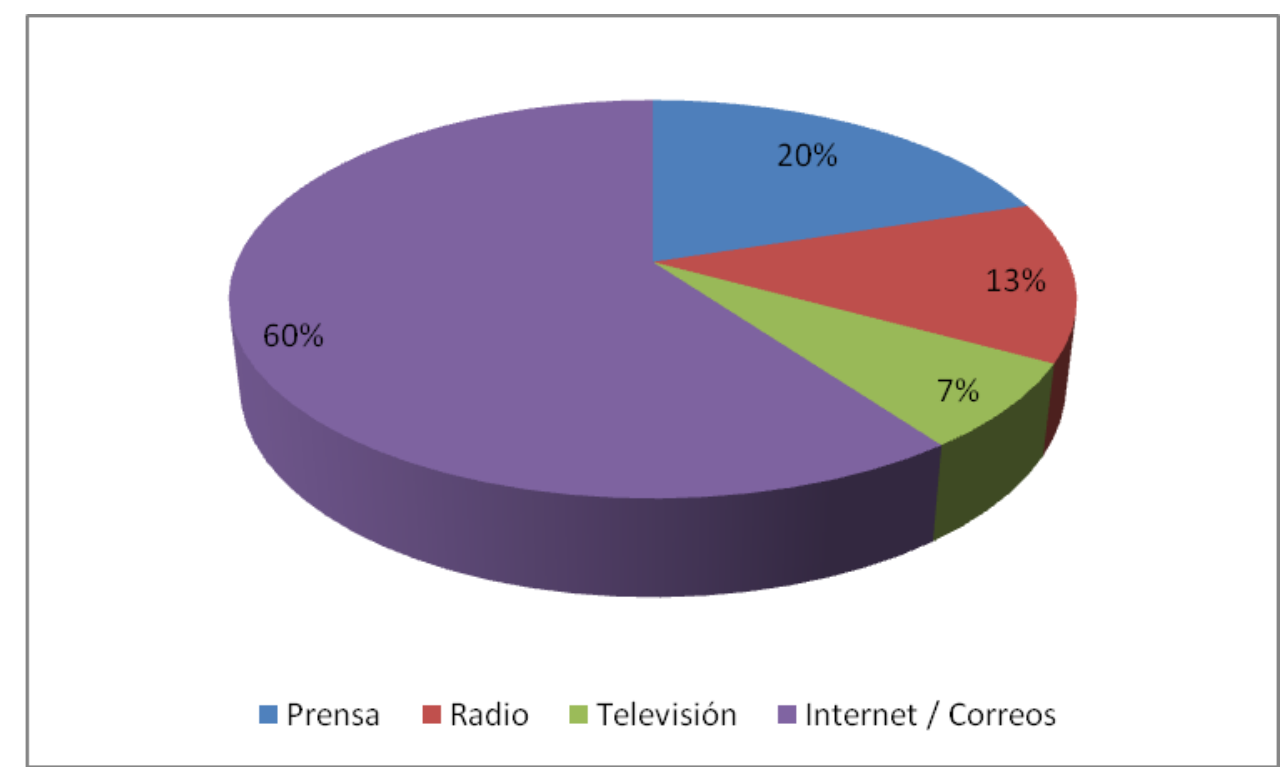

Fuente: Elaboración propia

\section{Gráfico 3.}

\section{Medios para enterarse de las Actividades}


Reflejando las respuestas Grafico 4, en cuanto a si el personal conoce los resultados de las actividades académicas que desarrolla la FCES, las respuestas revelan que el $63 \%$ no los conoce en contra de un $37 \%$ que si los conoce, por lo que la Facultad debe realizar un gran esfuerzo para que los mismos sean conocidos, dada la importancia que dichas actividades representan para los miembros de la comunidad de FCES.

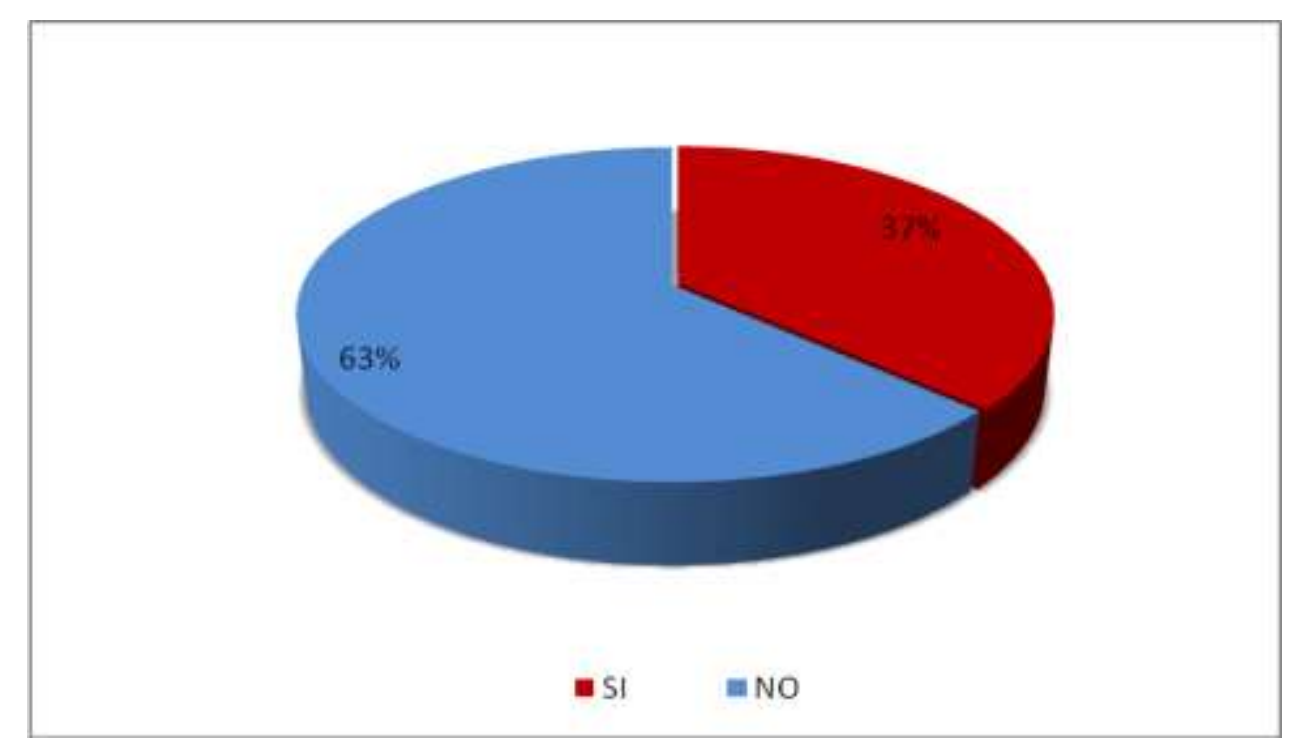

Fuente: Elaboración propia

\section{Gráfico 4.}

\section{Conocimiento de las Actividades Académicas}

Como lo plantea Garrido (ob.cit.), la implementación de una estrategia de comunicación corporativa será motor de cambios en los modos de gestión de las comunicaciones de la empresa, la cual se traduce en acciones, tácticas y campañas, que ponen en acción los propósitos planteados a nivel de diseño, por parte de los encargados de la administración y gestión de la comunicación en la empresa.

\section{CONCLUSIONES}

Una vez analizados e interpretados los resultados obtenidos, se puede establecer una serie de conclusiones basadas en los aspectos más significativos de la investigación, con respecto al objetivo del estudio relacionado con la "Imagen corporativa como herramienta de 
gestión institucional de la Facultad de Ciencias Económicas y sociales de la Universidad del Zulia.

Con relación a si la FCES da a conocer las actividades académicas que ofrece a la comunidad, se observó una alta presencia de la misma en el conocimiento de la comunidad, pero en el caso de si la forma en darse a conocer en la comunidad es clara y precisa el $53 \%$ reflejo ausencia, aspecto importante a tomar en cuenta.

En lo relativo, a como los medios de comunicación difunde información acerca de la FCES los resultados reflejan una alta presencia en los medios. Pero al analizar el aspecto relacionado, a si las autoridades de la Facultad, mantiene una comunicación directa con su personal, refleja una tendencia alta de ausencia en cuanto a la misma, lo que debe motivar a los directivos a reflexionar sobre este aspecto tan importante de comportamiento organizacional.

De igual manera, cuando se pregunta a través de cuales medios el personal académico se entera de las actividades que desarrolla la FCES, se puede evidenciar que el porcentaje más alto, reporta que es a través de la Internet, vía correos electrónicos, Esto evidencia que aun cuando la misma es un excelente medio para transmitir las actividades académicas, de investigación y extensión que lleva a cabo la Facultad, se hace necesario mayor presencia en los distintos medios de comunicación de manera que estas sean conocidas por toda la comunidad de la región. Para concluir la dimensión necesidades en materia de comunicación, en cuanto a si el personal conoce los resultados de las actividades académicas que desarrolla la FCES, las respuestas revelan que el $63 \%$ si la conoce, lo que representa un aspecto positivo en la gestión de la Facultad.

Las conclusiones obtenidas hacen pensar la necesidad creciente que tienen las instituciones de Educación Superior, en considerar la efectividad de planes de comunicación e imagen enmarcados en la comunicación corporativa, orientados a la promoción del quehacer universitario y su repercusión en los públicos internos y externos.

Es por ello, que se deben generar procesos comunicacionales que permitan proyectar la imagen corporativa de las instituciones universitarias siendo este uno de los elementos de relevancia estratégica a fin de garantizar el crecimiento de las organizaciones, para lo cual es prioritario tener una visión global de la comunicación de las instituciones universitarias y la relevancia que estas tienen en el desarrollo del país 


\section{REFERENCIAS}

Álvarez M., Mari y Maldonado, M. (2009). Utopía Universitaria. Revista Electrónica Educare. (Enero - Junio) Vol. 13 No 1. UPEL Universidad Pedagógica Experimental Libertador

Black, S. (2004). ABC de las Relaciones Públicas. Ediciones Gestión 2000, S.A.

Carrillo, A.(2011) La transformación universitaria desde la cultura del poder. Revista Educare, Volumen 15, Número 3, Septiembre-Diciembre , pp. 145-163. UPEL Universidad Pedagógica Experimental Libertador. Barquisimeto. Lara

Costa, J. (2003). Identidad Corporativa. Tercera Edición. México. Editorial Trillas, S.A.

Ferrer, J. y Pelekais, C. (2004). Tendencias Gerenciales y la Gestión Universitaria. Revista de Ciencias Sociales (RCS). Vol. X, No 1, Enero Abril, pp.148 - 163. FACES - LUZ

Garrido, F. (2007). Comunicación Estratégica. Las claves de la Comunicación Empresarial en el Siglo XXI. España. Ediciones Gestión 2000.

Gómez, C. y Villalobos, J. (1998). Como Gerenciar la Imagen Corporativa. Ediciones Plain Art, c.a. Caracas. Venezuela

Ivancevich, J., Lorenzi, P. y Skinner, S. (1997). Gestión, Calidad y Competitividad. España: Editorial Mc Graw - Hill.

Koontz, H. y Weihrich, H. (1998). Administración. Una perspectiva Global. Onceava Edición. México: Editorial Mc Graw - Hill

Madia de S., F. (1995). La Sexta Generación del Marketing. Santa Fe de Bogota, Colombia: Editorial Mc Graw-Hill.

Miquilena, E. (2002). Imagen Corporativa de Instituciones de Educación Superior según el Plan de Desarrollo Estratégico. Tesis Doctoral No publicada. Maracaibo: Universidad Dr. Rafael Belloso Chacín

Ochoa, E. (2004). La Comunicación en el Proceso de Transformación Universitaria. Tesis de grado para optar al título de Doctor en Ciencias Gerenciales. Maracaibo: Venezuela Universidad Dr. Rafael Belloso Chacín

Perozo, G. (2002). Imagen Corporativa de una Universidad a Distancia. Trabajo de Ascenso No publicado. Universidad Nacional Abierta. Venezuela

Pizzolante, I. (1999). Imagen y Comunicación. VI Programa de Gerencia de Comunicación e Imagen Corporativa. (Documento Inédito). Caracas. IESA.

Rincón, E., Graterol, M. y Díaz, Z. (2003). Imagen Corporativa sobre la Universidad del Zulia: Opinión y percepción de los líderes del Estado Zulia. Revista de Ciencias Sociales (RCS). Vol. IX, No 3, Septiembre - Diciembre. FACES - LUZ

Sáez, A. (2003). Estrategias de mercadeo para reposicionar la imagen institucional en los estudiantes de las Universidades Públicas Nacionales en el Municipio Maracaibo. Trabajo de Grado No Publicado. Universidad del Zulia 
Scheinsohn, D. (1998). Dinámica de la Comunicación y la Imagen Corporativa. Buenos Aires, Argentina. Fundación OSDE

Suárez, S. (2009). Gestión Comunicacional en las unidades de Información y Comunicación de la Universidad del Zulia. Trabajo de Grado no Publicado. Maestría en Ciencias de la E. División de Estudios para Graduados de la Facultad de Humanidades y Educación. Universidad del Zulia. Maracaibo, Venezuela

Valle, M. (2003). La Comunicación Organizacional de Cara al Siglo XXI. Razón y Palabra. Revista Electrónica en America latina Especializada en Comunicación. Abril - Mayo 2003. No 32. México

Van Riel, C. (1997) Comunicación Corporativa. Madrid, España: Editorial Prentice-Hall

Villafañe, J. (1999): Imagen positiva. Gestión estratégica de la imagen de las Empresas. Madrid: Ediciones Pirámide. 